\title{
THE DIAGNOSIS OF CEREBRAL ANGIOMA
}

\author{
By I. C. K. Mackenzie, M.D., M.R.C.P. \\ Chief Assistant, Department of Nervous Diseases, Guy's Hospital, London
}

The cerebral angioma is an arterio-venous communication of congenital origin and in its structure does not differ essentially from similar malformations elsewhere. Their occasional presence in the brain was already known to pathologists nearly 100 years ago and the first presumptive clinical diagnosis was made in 1898 . This was in a patient who had a left spastic hemiparesis and a loud bruit, audible over the whole head. Thereafter an increasing number of cases was found, in the early years of this century usually as a surprise finding at autopsy and later, with the development of neurosurgery, at operation for suspected cerebral tumour. Throughout these years the clinical picture was gradually taking shape. It is, however, with the development of arteriography and especially with the perfection of the percutaneous method within the last few years that the most recent and the most considerable advance in our knowledge of the clinical behaviour of these malformations has been made. Collapsed at autopsy and only partly exposed at operation, their structure has only been fully visualised and their true incidence appreciated by arteriography. Thus it now seems likely that all are composed both of arteries and veins, although in greatly varying proportion, and are arterio-venous malformations. They are of all sizes, the smallest being so small as possibly to escape notice by any other method of inspection. The incidence is about I per cent. of all neurosurgical and neurological admissions, males and females being about equally affected.

Although ultimately a radiological diagnosis, the clinical picture may at times be so well-defined as to allow of a confident diagnosis at the bed-side. In most cases, however, the diagnosis will be put forward on clinical grounds as a possibility, immediate or remote, and occasionally an angioma will be discovered by arteriography when quite unsuspected. It is the purpose of this paper to draw attention to clinical features of the cerebral angioma which might suggest the diagnosis and thereby also suggest arteriography as the radiological investigation of choice.

\section{Symptoms}

Although supposedly of congenital origin, the cerebral angioma does not immediately manifest itself and only about ro per cent. of patients harbouring one will have a relevant symptom in the first decade of life. More than half will have a presenting symptom in the next two decades and 70 per cent. will have had one before the age of 30 . The remaining 30 per cent. present with decreasing frequency during the next 20 years and presentation is extremely rare but not unknown over the age of 50. The ways in which angiomas commonly present are by causing haemorrhage, epilepsy, headache or hemiparesis and their further coursen may be characterized, if sufficiently prolonged, bye a combination of two or more of these symptoms

\section{Haemorrhage}

Angiomas frequently bleed and may do so in such a way as to present as a subarachnoid haemorrhage, with or without focal signs, or as an intracerebral haemorrhage, with or without blood in the subarachnoid space. There is nothing distinctive about the subarachnoid haemorrhage and in any individual case, where there are no focal signs, an angioma may be the source of the bleeding. Considering their relative frequency, an aneurysm is naturally a much more likely source and it is only the presence of certain signs that will allow one or other diagnosis to be favoured more than chance would suggest. Thus an accompanying third nerve palsy would clearly suggest aneurysm whereas a visual field defect suggests an angioma. An accompanying hemiparesis increases the possibility of angioma to the extent that angiomas are usually to be found in the cerebral substance whereas aneurysms predominate on the circle of Willis or close to it. This is not to say that a ruptured aneurysm cannot at times give rise to a profound hemiplegia.

The diagnosis of intracerebral haemorrhage will be entertained in the presence of a rapidly expanding intracranial lesion. The source of this can be an angioma and this is especially likely if the 


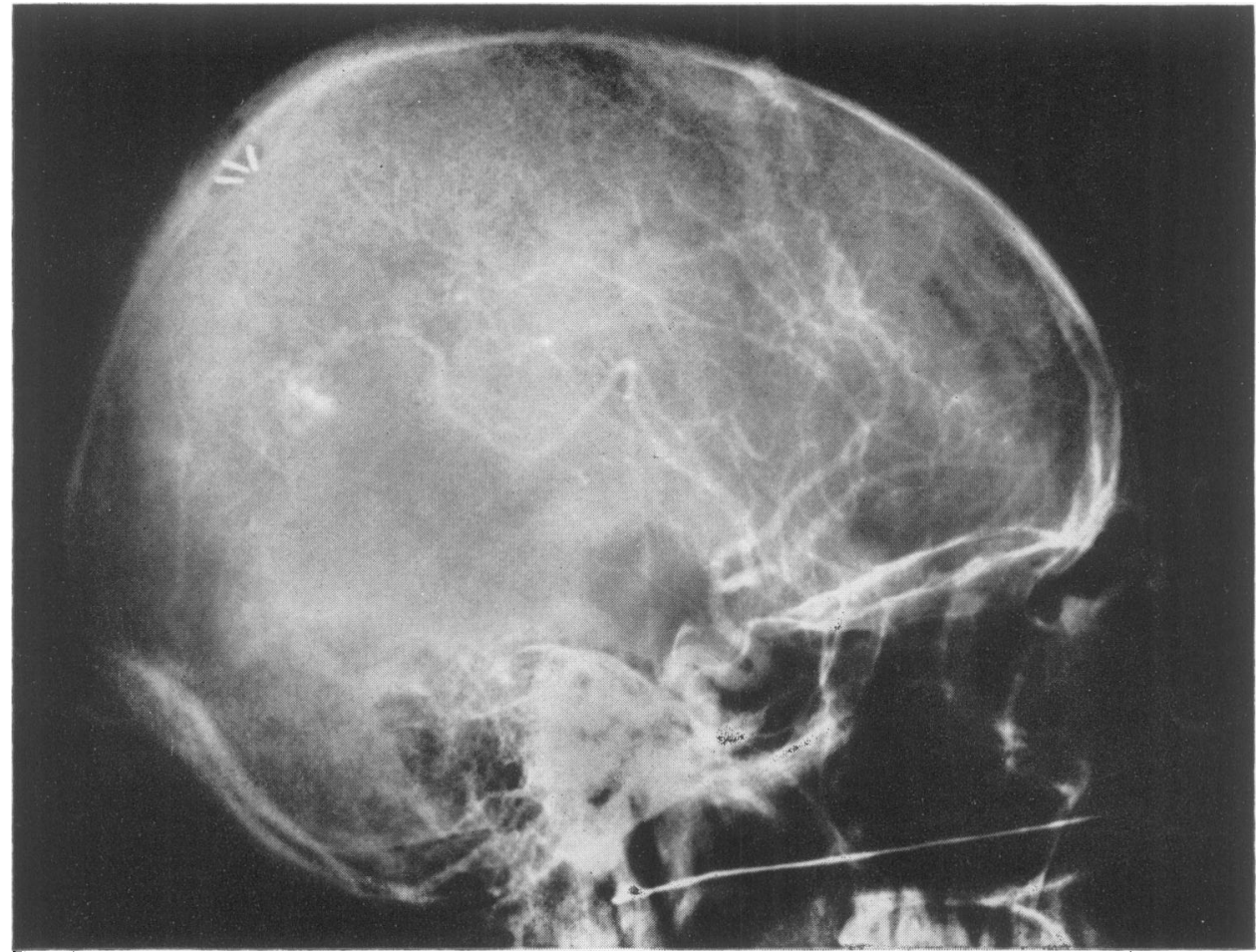

FIG. 1.-Right Carotid Arteriogram: This shows a small parietal angioma which antero-posterior views showed to be deeply situated. The arteriogram also indicates the presence of intracerebral clot. Subsequently this and the angioma were successfully removed.

patient is young, has a normal blood pressure and presents no other obvious cause. The following is an illustrative case:

E.P-B. complained one day, at the age of 18 , of severe aching in the right temple and vertex. Next day she was drowsy and dysphasic and continued to have severe right temporal and vertical headache spreading, but with slightly diminished intensity, over the whole head but not radiating into the neck. She started to vomit and complained now of diplopia and occasional blurring of vision.

Five days after the onset of headache she was found to have a complete left hemianopia, a moderate left hemiparesis including the face with slightly increased jerks and no sensory loss. She was left-handed. There was no bruit.

Ten days from the onset of the headache she developed papilloedema.

The C.S.F. was under slightly increased pressure but was normal in composition.

Arteriography demonstrated a small angioma in the right parietal region. (Fig. I).

\section{Epilepsy}

A cerebral angioma presenting with epilepsy may do so with a generalized seizure, preceded by a brief warning or none at all, but it is likely that some focal feature will develop as the attacks recur. The attacks may, from the outset, have a clear focal signature. This may lead into a generalized seizure or the attacks may remain focal. The two may alternate, as the following case illustrates:

R.P., aged 25, had his first attack I I years previously when a twitching suddenly appeared in the right little finger and rapidly involved the other fingers. There was no further spread and the attack was over in a few minutes. Six months later he had another attack and on this occasion the right hand became dead before the clonic movements started. Again he had freedom for six months when a similar attack developed and since then he has had many attacks which started either in the right little finger or right thumb, spread to the other fingers, the wrist, the arm and sometimes to the chest and abdomen. Twice when the clonic movements had spread to involve the right chest and abdominal muscles, the right side of the face and tongue became numbed and were the seat of pins and needles. These sensations persisted for the duration of the attack only 


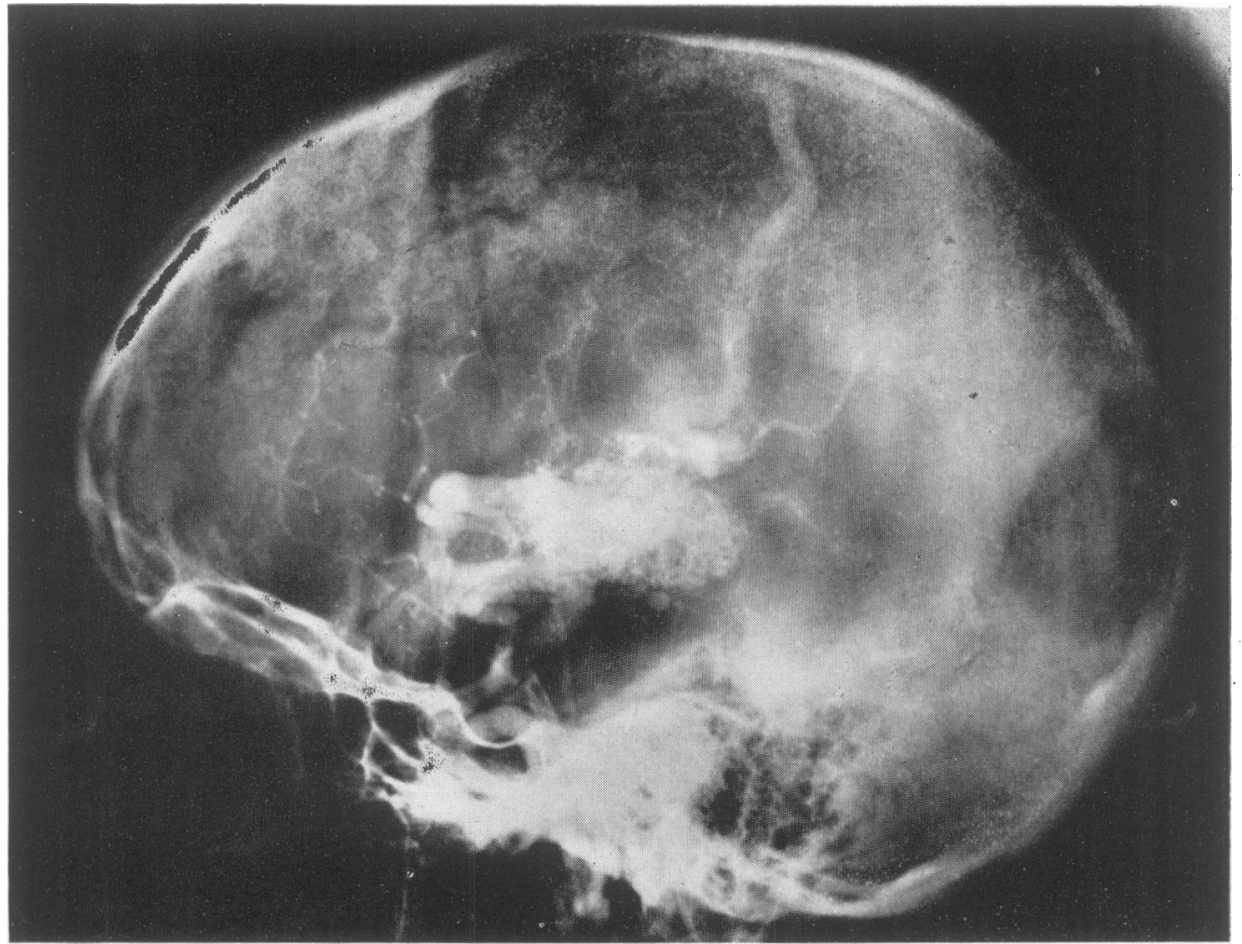

FIg. 2.-Left Carotid Arteriogram: This shows an angioma in the left Sylvian region. There is also filling of large draining veins when the contrast is still visible in the carotid artery, evidence of an arterio-venous shunt.

and were accompanied by an inability to speak. After such an attack he would have a transient weakness of the right arm, lasting 10-20 minutes, without accompanying sensory symptoms.

Three years after the initial attack, an episode starting in the usual way but of particular severity, led to loss of senses and a generalized convulsion. In the following years he had had about ${ }_{15}$ such attacks intermingled with the lesser ones.

For six years he had had a permanent slight weakness and clumsiness of the right hand and for three years a slight weakness of the right leg.

On examination he had mild papilloedema, a slight right hemiparesis and an intracranial bruit.

The focal nature of an epileptic attack must inevitably suggest the presence of some demonstrable cause but the attacks are not always so suggestive of a gross lesion as the ones just described nor are there necessarily accompanying physical signs in the nervous system to support such a suggestion. The following case illustrates another form of epileptic attack for which an angioma was responsible:

A.J., aged 23, had minor epileptic attacks since the age of 16 . The first occurred when she sat at work when suddenly something came over her head, everything sounded queer and the feeling passed off. The attack had lasted a couple of seconds and immediately she felt well.

For the next five years she repeatedly experienced such attacks, sometimes as often as three times in one day. They were always the same - ' a peculiar feeling passed over her head and everything sounded strange and distant.' At times she also noticed flushing of the face accompanied by a burning sensation. She never told anyone about these episodes and her mother reported that during these five years nothing abnormal was ever observed.

Fifteen months before the diagnosis of angioma was made she had the first of two major attacks. She suddenly felt queer, as with a minor attack, and everything seemed to go dim. When asked what was the matter she could not reply and uttered only a few unintelligible sounds. This premonitory period lated about two minutes and then she lost her senses. She recovered about 20 minutes later but could not speak properly for an hour.

Thereafter minor attacks continued about once daily and now, on two occasions, she noticed some speech difficulty during the minor attacks. 


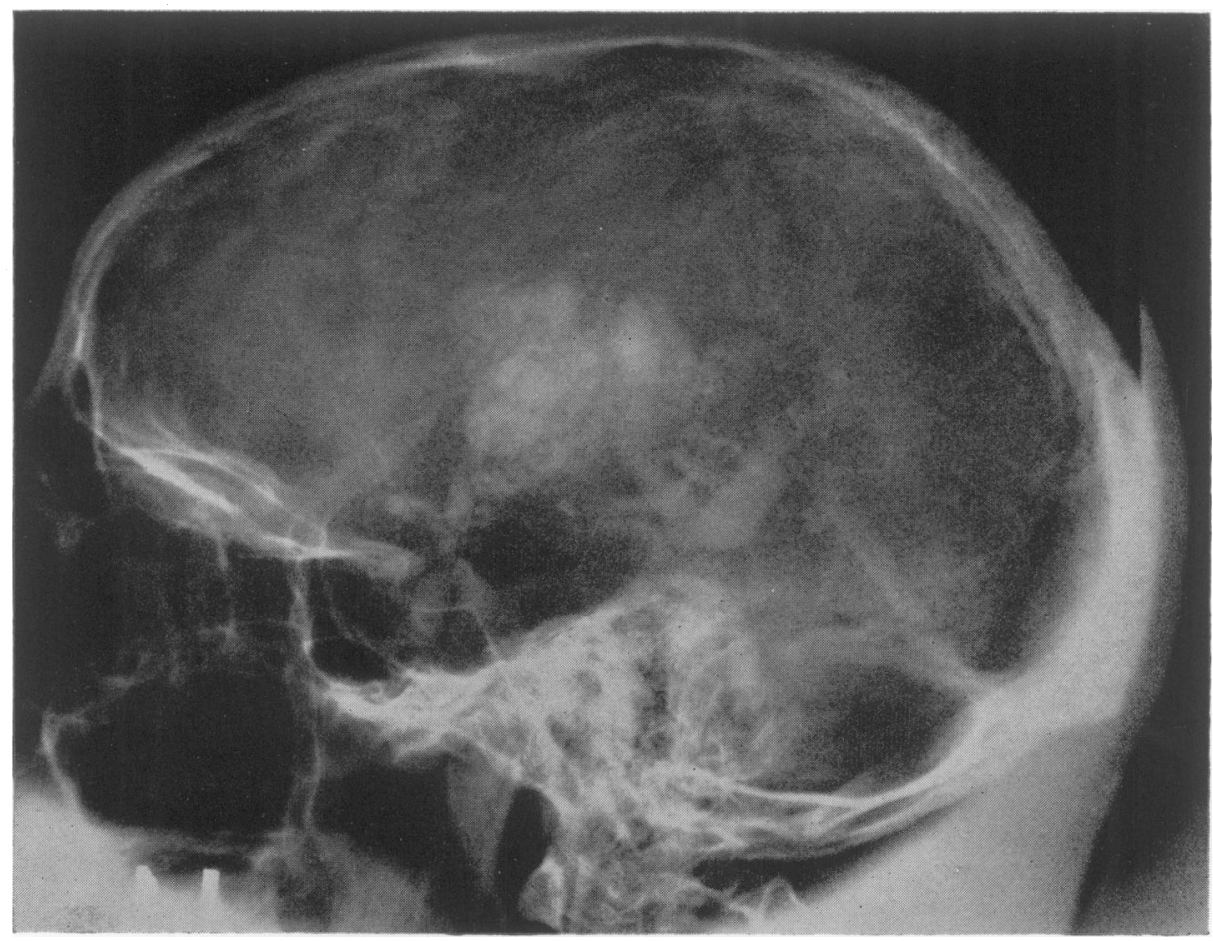

FIGS. 3 and 4.-Left Carotid Arteriogram: This shows a large deeply-situated fronto-parietal angioma, with early filling of draining veins entering the straight sinus. Anterior posterior view on next page (Fig. 4).

Four months before coming under observation she had a second major attack similar to the first.

On examination there were no abnormal signs apart from a bruit heard over the left orbit. Arteriography showed an angioma in and around the left Sylvian fissure. (Fig. 2).

To sum up, epileptic attacks which are to be attributed to an angioma may be expected at some time to demonstrate their focal nature. They may show variability in an individual patient, as well as in themselves, and a widely varying periodicity. Patients in whom an angioma has already caused one or more epileptic attacks may then have freedom from attacks for several years.

\section{Headache}

Some patients harbouring a cerebral angioma suffer predominantly from headache. When not the leading symptom, headache does not play a prominent part in the symptomatology although brief headache occasionally develops following an epileptic attack. No reference is, of course, being made to headache in association with haemorrhage.

Usually intermittent and severe, the headache and associated symptoms are frequently suggestive of migraine. It will be found however that whereas in constitutional migraine the attacks tend to vary in their symptomatology, in cases of cerebral angioma the migrainous attacks tend to follow the same pattern. The aura, whether visual, somatic sensory or motor, will remain fairly constant in its nature and distribution. The subsequent headache, if localized, will always be in the same situation and sometimes the normal sequence of an aura being replaced by headache will be disturbed. The following rather unusual case illustrates these points:

E.S. developed, for the first time at about the age of 12 , a dull headache in the left frontal region which was followed in a few minutes by weakness of the right arm and right leg. She also lost the ability to speak. There was no impairment of consciousness and she did not fall. After about an hour the attack would pass off with a sensation of ' lights' passing across her field of vision. At first the attacks were infrequent but gradually increased in frequency so that, within two years or so, she was having one every month or two. All had exactly the same pattern. They then became less frequent again until the age of 18 when she had the last attack of this nature.

Four years later she had a convulsion with loss of senses, preceded by an inability to speak, and two similar attacks in the course of the next 15 


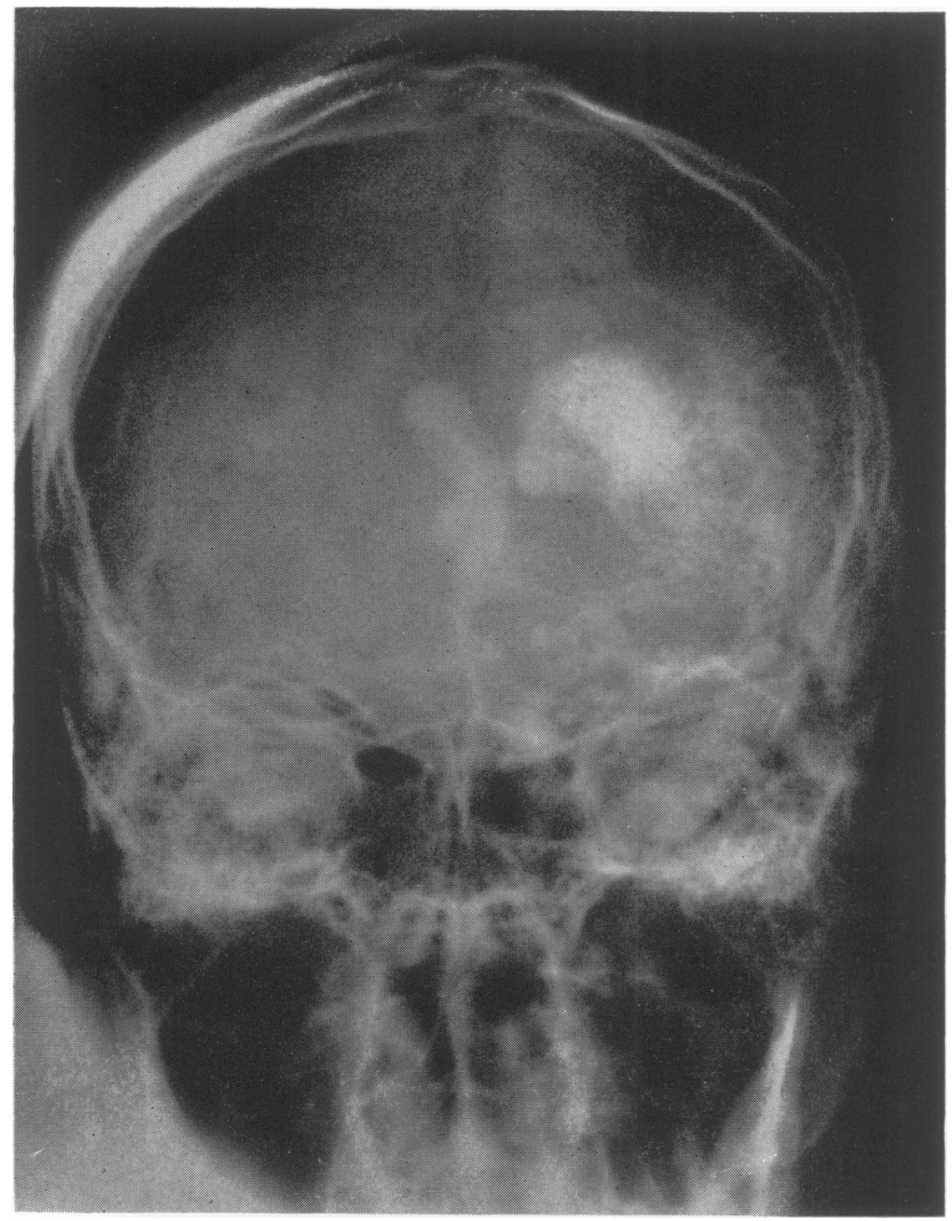

FIG. 4.

months at the end of which time the diagnosis of angioma was made.

On examination at that time she had minimal right-sided signs and a bruit which could be heard over the left temporal region.

Arteriography showed a left frontal angioma.

This case also demonstrates the tendency for the migraine to be followed by epilepsy but this, as well as the other features to which attention has been drawn, occurs from time to time in migraine for which no demonstrable cause can be found.

\section{Hemiparesis}

Occasionally, in the absence of haemorrhage or epilepsy, hemiparesis develops. Sometimes this occurs in an episodic manner but sometimes gradually, as the following case illustrates:

T.C., aged 27, had suffered from loss of power in the right hand for four years. This began with a lack of facility in fine finger movement and he first became aware of this when he experienced some difficulty in playing the accordion which he normally did with professional expertness. He gradually deteriorated to such an extent that he had to give up playing the accordion. Two years after he first noticed the weakness he became clumsy in the performance of coarser tasks and, in his job as a motor mechanic, he experienced difficulty in holding a hammer and in the use of a screw-driver. As the weakness gradually increased he tended to do most things with his left hand. During the last two years he noted a tendency to take a longer step with the left foot and started to kick with his left foot when playing football. Clonus would develop at the right ankle while dancing.

There was no history of headache, vomiting, visual disturbance or epilepsy.

On examination he had a right spastic hemiparesis with slight impairment of two-point discrimination in the right hand and foot. An 
intracranial bruit was found on auscultation over the left orbit and temporal fossa.

Arteriography revealed a large deeply situated fronto-parietal angioma. (Figs. 3 and 4 ).

Most cases of angioma will present with haemorrhage, epilepsy, headache or hemiparesis. Other presentations which have been recorded include symptoms of raised intracranial pressure with papilloedema and without any of the symptoms already mentioned, unilateral pulsating exophthalmos and in one case the presentation was with unilateral blindness with bilateral optic atrophy.

\section{Additional Symptoms}

Any symptom with which an angioma may present is one shared with various other pathological conditions. The diagnosis of angioma becomes more likely when certain additional symptoms occur. Of these by far the most significant is haemorrhage, where that has not been the presenting symptom and, indeed, oftrepeated subarachnoid haemorrhage is in itself quite suggestive. Thus if a patient with a history of epilepsy or of intermittent headache of a migrainous type or with a hemiparesis has a subarachnoid haemorrhage, the chances of an angioma being the cause are greatly increased. It was observed earlier that an aneurysm is a much more likely source of haemorrhage but an unruptured aneurysm seldom gives rise to epilepsy, migraine or hemiparesis.

Many patients with an angioma develop epilepsy when this has not been the presenting symptom. Mention has already been made of patients with atypical migraine later developing epilepsy and this has also been observed in patients whose first symptom has apparently been hemiparesis. It also occurs, however, after haemorrhage which has been apparently subarachnoid, indicating that the bleeding has been, at any rate in part, intracerebral. This conclusion increases the likelihood of the source of the haemorrhage being an angioma, situated as it usually is within the cerebral substance. The following is an illustrative case:

H.B-S., aged 36 , awoke one morning six months before coming under observation, with a severe fronto-occipital headache. While sitting on the edge of a sofa she vomited and then 'fainted.' She came round six hours later still with the same headache, which persisted and was aggravated by bending. For two to three days she could not keep any food down. A week after this incident a diagnosis of subarachnoid haemorrhage was established by lumbar puncture.

Two months later she lost her senses without warning and had a succession of fits. These were observed to start in the left hand but became rapidly generalized with the head and eyes turned to the left.

On examination subsequently there were noc abnormal signs in the nervous system and there was no bruit. The B.P. was $130 / 80$.

Arteriography demonstrated a small angioma in the right frontal region.

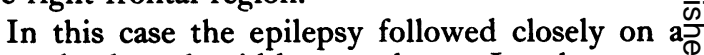
proved subarachnoid haemorrhage. In other cases the relationship may not be so close and theis haemorrhage not so definite. In this connectionit is important to realise that the true nature of $a^{\circ}$ subarachnoid haemorrhage is often overlooked and $\vec{\omega}$ that it may masquerade, amongst other things, as an unproved meningitis, a threatened meningitis, fibrositis of the neck and, in this country, sunstroke Enquiry into past symptoms must be sufficiently. comprehensive to include incidents of this nature.

\section{Signs}

Abnormal signs are present in about 70 per cent.o of cases of angioma. The common finding is a spastic mono- or hemiparesis with appropriated reflex changes and with or without sensory impairment. When present the sensory impairment? is usually of the so-called cortical type. Son times the signs are confined to a defect in the visigil fields. Taken by themselves the signs are of lifieco value except in localizing the lesion, which they with accuracy, and it is only in relation to the nature of their onset and subsequent course that abnormal signs in the nervous system become significantly suggestive of an angioma. Perhapso the most helpful are those which develop in relation to migrainous attacks and may be confined to defects in the visual fields. This has been? recorded in ordinary migraine but must suggest the possibility of an angioma whose presence can only be excluded by doing not only carotid but also vertebral arteriography. Similarly the much? rarer development of a hemiparesis in relation tomigrainous attacks must suggest the diagnosis of angioma.

Except in relation to haemorrhage papilloedemas is infrequent but has been occasionally observedo It has already been mentioned as one of the rarer? methods of presentation.

The intracranial bruit is a sign to which greatsignificance has rightly been attached but the estimated incidence of which has varied greatlyo throughout the years. At first regarded as a sinen qua non it soon became apparent that not alf angiomas were associated with a bruit and a few years ago a series of more than 60 angiomas was? reported in which the incidence of bruit was onlye 8 per cent. The present writer recently reviewed a series of 50 angiomas in which a bruit was 


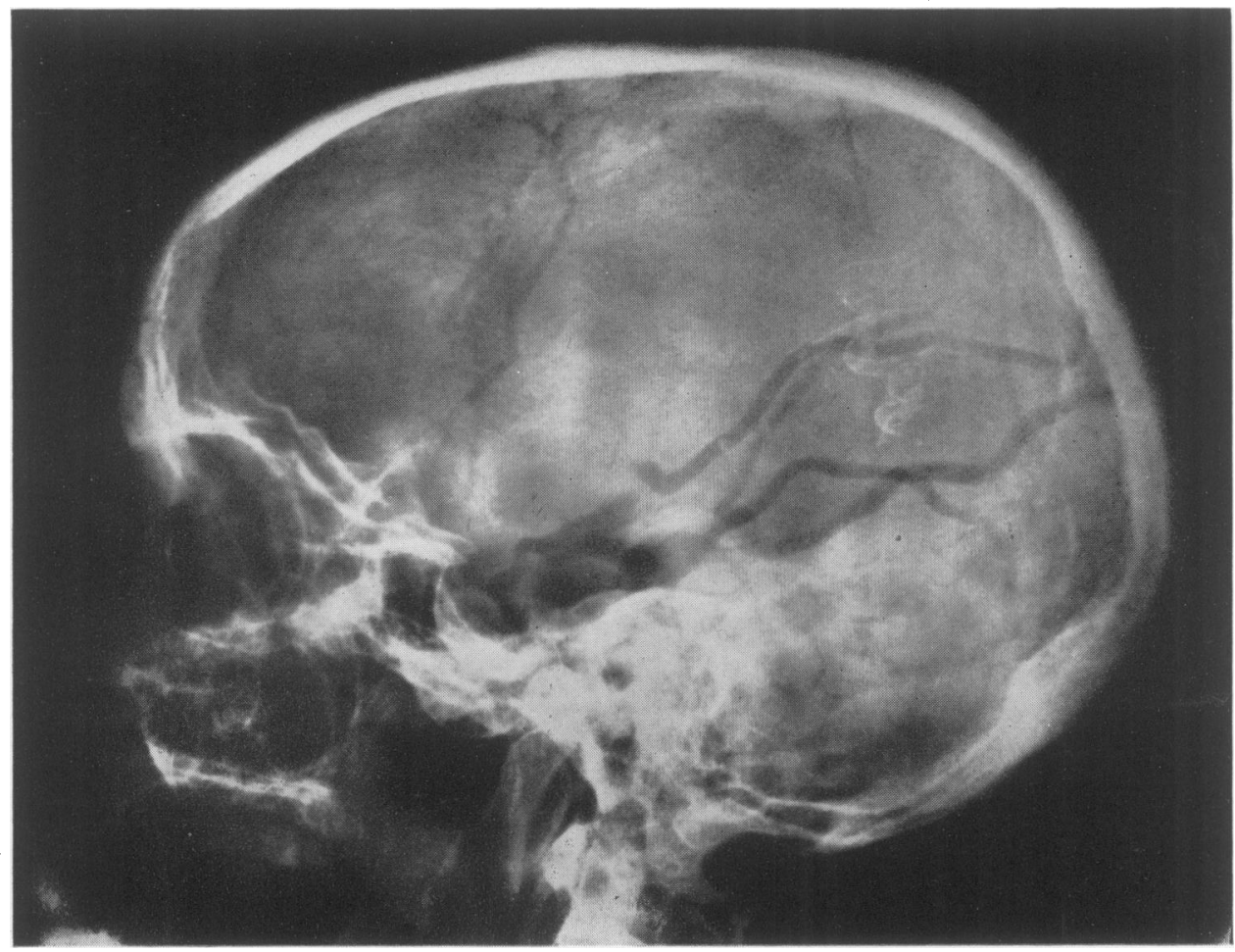

FIG. 5.-Lateral X-ray of Skull: This shows enlargement and tortuosity of the vascular channels. Characteristic vascular calcification is present in the left parietal region.

detected in 50 per cent., this finding however being rare in patients whose presenting symptom was that of haemorrhage. In the remaining cases it seemed therefore a sign of diagnostic importance. It is, of course, sometimes faint and occasionally intermittent and, although frequently heard over the skull, cannot be regarded as absent until auscultation over the eyeballs in quiet surroundings has failed to detect it. A bruit is not pathognomonic of angioma but in cases where the symptoms and signs, if any, are otherwise suggestive, the finding of a bruit must be accorded the greatest significance. In the writer's experience the failure to detect a bruit in patients whose symptoms, apart from haemorrhage, suggest the possibility of angioma, is a point against the diagnosis. The six patients to whose case records reference has been made were selected in order to illustrate characteristic symptoms rather than signs. It will be seen, however, that a bruit was present in four and that in the other two the presenting symptom was haemorrhage.

Earlier observations on the effects of an arteriovenous shunt on the circulation and its tendency to cause dementia are no longer of any diagnostic importance.
It is rare for the blood pressure to be abnormal.

\section{Angiomas in the Posterior Fossa}

So far only supratentorial angiomas have been considered. Their occurrence in the posterior fossa is much rarer as might be expected from the lesser amount of vascular tree in the posterior fossa which could be the seat of malformation. The impression that they are even rarer than this explanation would allow may depend on the fact that vertebral arteriography is not yet performed with the same facility as carotid arteriography and thus some angiomas in the posterior fossa are not being discovered.

The modes of presentation of angiomas in the posterior fossa are naturally restricted by their localization and they seem to present either with haemorrhage or as a posterior fossa space-occupying lesion. In one case, remitting symptoms and signs of brain-stem involvement had led to a diagnosis of disseminated sclerosis which had been sustained for some years and, in fact, until auscultation revealed a loud bruit. As with supratentorial lesions which suggest angioma, the bruit is a valuable sign in posterior fossa angiomas but not, of course, pathognomonic. 


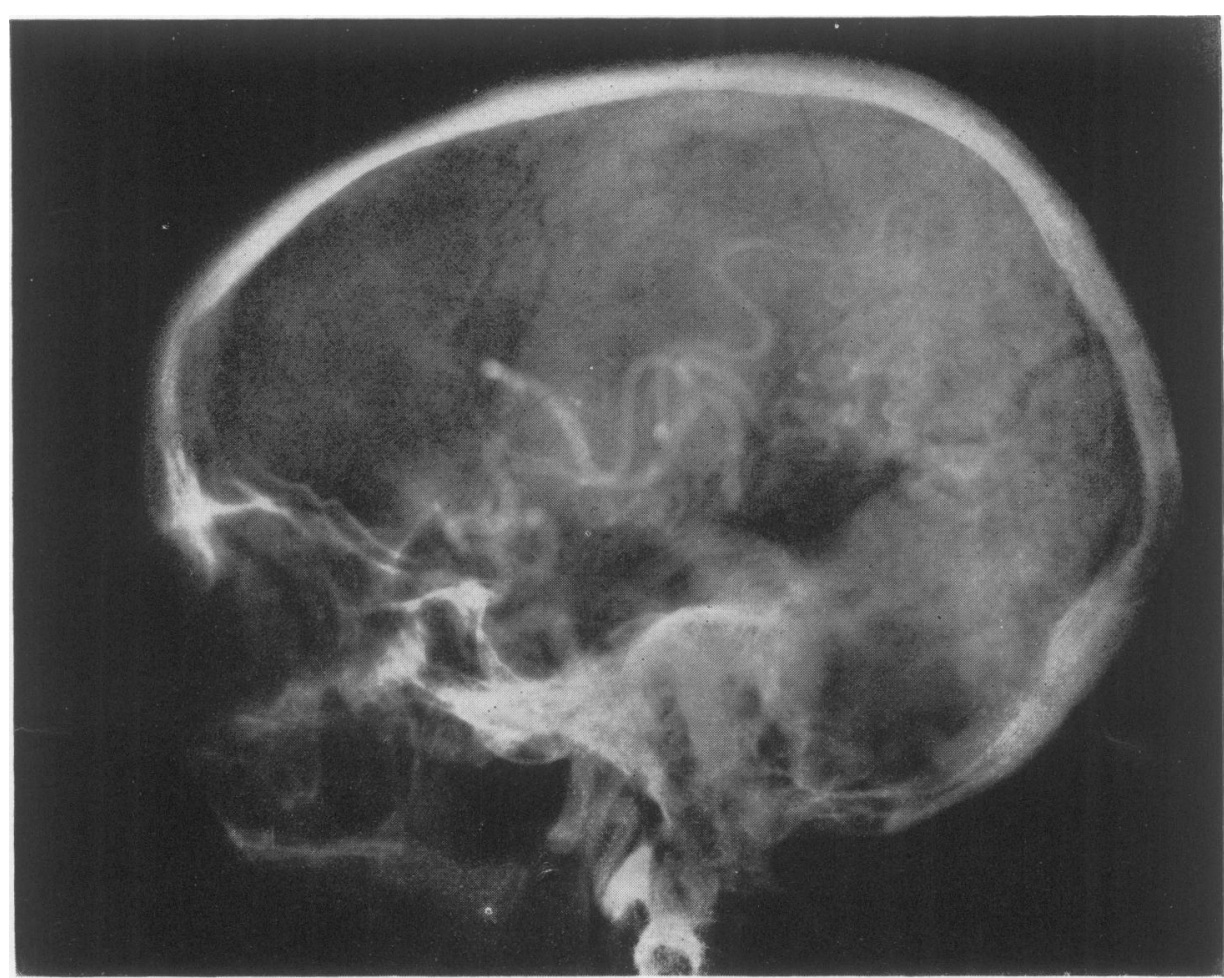

Fig. 6.-Left Carotid Arteriogram: This shows contrast passing through enlarged branches of the middle cerebral artery and early filling of what later films showed to be an enormous parieto-occipital angioma. This angioma did not manifest itself until the sixth decade.

\section{Investigations}

Lumbar Puncture. Except in the rare cases of angioma which act as a space-occupying lesion the C.S.F. is under normal pressure. It is always normal in composition unless the angioma has ruptured when there may be blood in the subarachnoid space. If the haemorrhage is purely intracerebral the C.S.F. may be normal or may show a cellular reaction with increased protein.

Skull $X$-ray. X-ray of the skull is usually normal. If changes are present they may consist of enlargement of the carotid canals and an increased asymmetrical vascularity of the vault. Calcification may be present at the site of the angioma. (Figs. 5 and 6 ).

If the angioma is in the posterior fossa the channels traversed by the vertebral artery may be enlarged.

Electroencephalogram. In contrast with spaceoccupying lesions the E.E.G. may be normal in about half the cases. This observation applies, of course, only to the cases in which there has been no recent intracerebral haemorrhage which acts in this respect, as in others, as a space-occupying lesion. When abnormalities are present they may be over the angioma but are more often around ito sometimes in the form of slow or sharp waves and/or spikes. More rarely there is a diminution of the amplitude on the ipsi-lateral side of the record. In some very large angiomas the E.E.G. has been apparently normal.

\section{Diagnosis}

Cerebral angiomas give rise to symptoms from. the early years to late in life but, for the first time most often between the ages of 10 and 30 yearsi The initial symptoms are likely to be haemorrhage epilepsy, intermittent headache or hemiparesis The relative frequency of each mode of presenta? tion is not yet established but it is possible that haemorrhage will be found to be an increasingly. frequent presentation. Not the initial symptono alone but the combination, as the clinical picture develops, of two or more of those mentioned sugro gests angioma. The significance of haemorrhage at any stage in the clinical history of a patient suspected of having an angioma has beef emphasised.

Angiomas are of all sizes but whereas any angioma may bleed, it is only by virtue of 
haemorrhage that the smallest can make their presence known. This has some bearing on the observation that angiomas whose initial symptom is haemorrhage seldom give rise to a bruit. These cases apart, a bruit is a valuable sign but although the chances of its being present increase with the size of the angioma it is sometimes absent with very large ones. If there has been no recent rupture, lumbar puncture is normal. X-rays of the skull and the E.E.G. are often normal. It is on the careful analysis of symptoms that the decision whether or not to perform arteriography will depend and this is the crucial investigation.

I am grateful to Sir Charles Symonds, Dr. J. St. C. Elkington, Mr. Wylie McKissock, and Mr. Murray Falconer'for permission to quote from the case records of patients under their care.

The X-rays were taken in the Departments of Radiology in the National Hospital, Queen Square, and in the Guy's-Maudsley Neuro-Surgical Unit.

The photographs were made in the Photographic Department, Guy's Hospital.

\section{PULMONARY TUBERCULOSIS}

\section{(Postgraduate Medical Journal)}

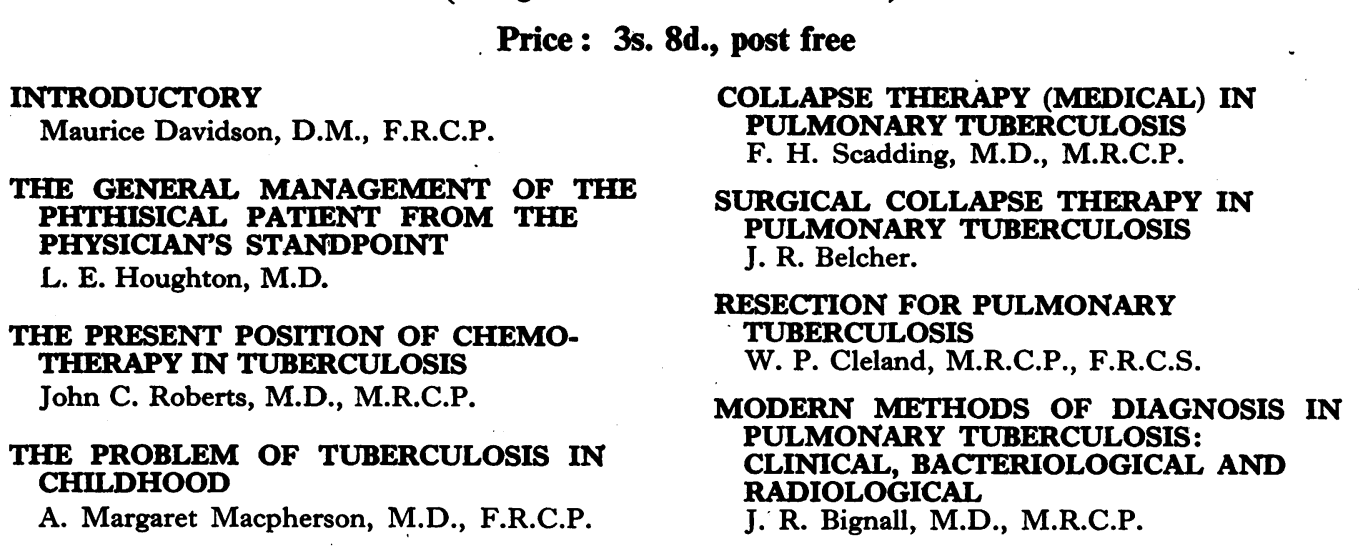

Published by

THE FELLOWSHIP OF POSTGRADUATE MEDICINE

60, Portland Place, London, W.1

\section{BATTERSEA CLINIC FOR VARICOSE ULCERS}

A clinic for the study and treatment of varicose ulcers was opened by Sir Arthur Porritt, Sergeant Surgeon to the Queen, on October 20, at Battersea Central Mission. This Mission has for many years had a physiotherapy clinic, also a clinic for the treatment of varicose veins. This new clinic, in charge of Dr. Stanley Rivlin, is designed not only to treat but to provide for the aftercare of these patients. By careful re-examination and recording over a period of years it is hoped to amass a body of information on a subject hitherto largely neglected. One of the features of the clinic is provision for stereoscopic or tridimensional colour photography for record purposes before and after treatment. The patients are instructed to report at intervals after treatment. 